\title{
Diversity, abundance and seasonality of birds in a Caribbean pine plantation and native broad-leaved forest at Trinidad, West Indies
}

\author{
FLOYD E. HAYES and ISHMAELANGELO SAMAD
}

\begin{abstract}
Summary
Monoculture plantations of exotic Caribbean pine Pinus caribaea have been planted extensively in many tropical countries, but little is known about the bird communities inhabiting them. From October 1995 to September 1996, we compared bird populations in a $37^{\text {-ha }}$ stand of mature Caribbean pine forest (planted in 1972) with an adjacent, similar-sized stand of native broad-leaved forest at Mount Saint Benedict, Trinidad, West Indies. Ten censuses, each including to fixed-radius point counts $(25 \mathrm{~m}$ radius, 10 minutes duration), were conducted simultaneously during different months of the year in each habitat. Both species richness and species diversity were significantly lower in the pine forest, but the overall abundance of individual birds did not differ between the two forests. Seasonal variation in bird populations was more pronounced in the pine forest, where fewer birds (and presumably food resources) were present during the dry season. The pine forest bird community was dominated by forest edge specialists and the native forest by forest interior specialists at both the individual and species levels. At the individual level, nectarivores, aerial foragers and understorey foragers were proportionately more common in the pine forest; insectivores and canopy foragers were more common in the native forest. At the species level, more aerial foragers occurred in the pine forest and more understorey foragers in the native forest. The diversity of bird communities in pine plantations may be promoted by increasing habitat heterogeneity.
\end{abstract}

\section{Introduction}

The accelerating demands for energy, forestry products and space for grazing, agriculture and urbanization have fuelled deforestation in tropical regions, thus increasing the need to restore degraded lands into productive forests through natural regeneration or plantation forestry (e.g. Evans 1982). As a consequence, the establishment of secondary forest plantations has increased at an exponential rate; by 1981, such forests comprised more than $1 \%$ of tropical forest biomass (e.g. Brown et al. 1986). In spite of this ominous trend, ecological studies continue to focus primarily upon mature ecosystems (e.g. Brown and Lugo 1990). A better understanding of secondary forest ecosystems - including relatively "simple" plantation forest ecosystems - is vital if we are to manage tropical forests successfully in the future (Brown and Lugo 1990, Lugo 1992).

The ecology of bird populations inhabiting secondary forest plantations in the Neotropics remains poorly studied. The bulk of such studies have concentrated 
upon the ecology of birds inhabiting coffee Coffea arabica plantations (e.g. Perfecto et al. 1996, Wunderle and Latta 1996). Other secondary forest plantations such as mahogany Swietenia macrophylla (Cruz 1987) and Caribbean pine Pinus caribaea (Falkenberg et al. 1983, Cruz 1988) have received scant attention. The bird species inhabiting primary forests generally have more restricted geographical ranges than species inhabiting secondary forests and are presumably more prone to extinction (Wiedenfeld 1991). Identifying which species can and cannot exploit the resources available in plantation forests should be a high priority for conservationists and land managers, particularly since secondary forest plantations may provide refugia for forest birds in regions subjected to extreme deforestation (Brash 1987).

The Caribbean pine is the only member of its genus that is completely tropical in its distribution, with disjunct populations occurring in the Bahamas (var. bahamensis), western Cuba and the Isle of Pines (var. caribaea), and along the Caribbean seaboard of Central America from southern Mexico to Nicaragua (var. hondurensis; e.g. Perry 1991, Vidakovic 1991). Because of its high growth rate and ability to thrive in nutrient-poor soils of low altitudes, it has been planted in many tropical countries during the last several decades for its commercially valuable timber (e.g. Evans 1982, Anoruo and Berlyn 1993).

Monoculture stands of exotic Caribbean pine (var. hondurensis) have been planted extensively in the Northern Range and in other parts of Trinidad, West Indies, with an estimated 4,143 ha under production in 1987 (Lackhan 1972, 1973, Anon. 1989, Jhilmit 1992). Trinidad's total land area is 482,500 ha (Comeau 1991); thus, with an annual planting programme of 50-150 ha (Anon. 1989), approximately $1 \%$ of the island was covered with pine plantations by 1995 . Because the establishment of pine plantations usually requires the complete removal and burning of the native vegetation before planting, and the subsequent removal of secondary plant growth during the first 10 years (Lackhan 1972, 1973, Jhilmit 1992), the short-term effects upon the native fauna and flora must be devastating. But because of the fast rate of growth by pines, which is an estimated 15-20 times higher than that of native tree species, relatively tall (> $20-\mathrm{m}$ ) stands of mature pine forest may be present within 20 years (Lackhan 1972, 1973, Jhilmit 1992).

The establishment of monoculture Caribbean pine plantations in Trinidad and other tropical countries disrupts the complex relationships of the native forest ecosystems and decreases the diversity of plant and animal life (e.g. Torres et al. 1978, Bandeira and Souza 1982, Lugo 1992). However, there have been few studies of the impact of exotic Caribbean pine plantations on avian communities. During a brief study in Jamaica, Falkenberg et al. (1983) found fewer birds in Caribbean pine plantations than in native primary and secondary forest sites. Cruz (1988) studied the foraging behaviour of birds in a Caribbean pine plantation in Puerto Rico, but did not compare bird communities between the pine and native broad-leaved forests.

The purpose of the present study was to compare the diversity, abundance and seasonality of birds in a Caribbean pine plantation and native broad-leaved forest in Trinidad, and to compare the ecological characteristics (habitat, diet and foraging strata) of the two bird communities. Such comparisons may enable us to predict the kinds of changes that may take place in bird communities as native 


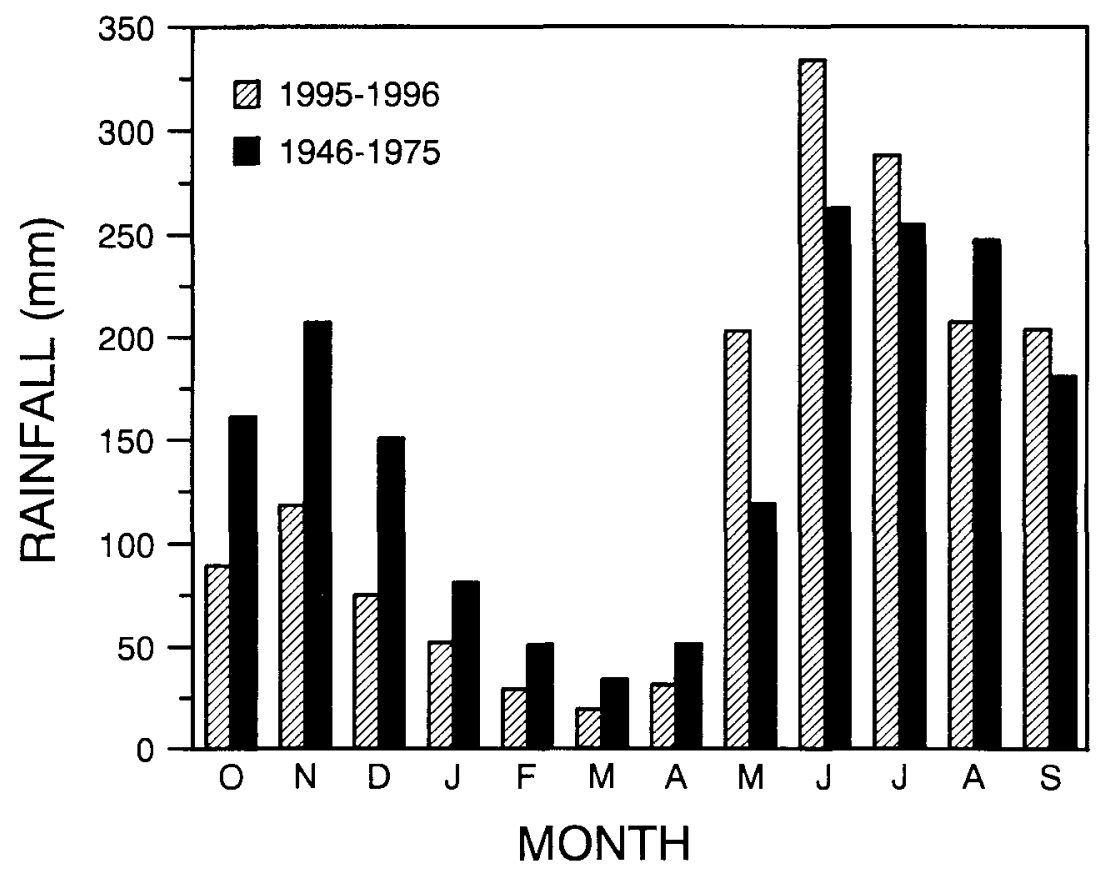

Figure 1. Monthly rainfall (mm) during the study period (October 1995 to September 1996) compared with monthly means for 30 years (1946-1975; Berridge 1981) at nearby Piarco, Trinidad.

broad-leaved forests are converted into monocultural pine plantations in Trinidad and other tropical countries.

\section{Study area}

Located off the north-eastern coast of South America, Trinidad is a large continental island with an impoverished but relatively harmonic avifauna in comparison with that of the adjacent mainland (Graves and Gotelli 1983, Snow 1985, Gotelli and Graves 1990, ffrench 1991). The climate of Trinidad is characterized by little seasonal variation in temperature and marked seasonal variation in precipitation, with a rainy season extending from late May through December and a dry season from January to late May (Figure 1); the amount of precipitation and the onset of each season varies greatly from year to year (Berridge 1981).

We studied bird populations in adjacent, similar-sized stands of pine forest and native broad-leaved forest on the southern slopes of the Northern Range at Mount Saint Benedict, Trinidad $\left(10^{\circ} 40^{\prime} \mathrm{N}, 61^{\circ} 24^{\prime} \mathrm{W}\right)$. The elevations of the study sites were comparable, ranging from 225 to $425 \mathrm{~m}$ (Trinidad and Tobago Government 1970, 1978), as were their edaphic and climatic conditions (no available data). The study sites were selected for their ease of access along established trails just above the Mount Saint Benedict monastery buildings (ffrench and Bacon 1992, Murphy 1995, Ramsawak 1996). Rainfall data from the 
study period were obtained from the nearest available station at Piarco, located $12.1 \mathrm{~km}$ to the south-east, and compared with monthly means from a 30 -year period (1946-1975; Berridge 1981) at the same locality (Figure 1).

\section{Pine plantation}

The pine plantation was planted in 1972 (according to a sign posted by the Forestry Division) and encompassed 37 ha (planimetric area measured from topographic maps; Trinidad and Tobago Government 1970, 1978). The forest was dominated by mature, cone-bearing pines up to $21+\mathrm{m}$ tall. Epiphytic plants were absent from the pine trees. The understorey vegetation comprised a thick layer of sedges (primarily Scleria bracteata) and other herbaceous plants up to $2 \mathrm{~m}$ high during the wet season, but most of the understorey vegetation withered and flattened as the dry season progressed. Scattered palms, dicot shrubs and broad-leaved trees occurred in some areas, particularly in the more humid ravines, but seldom extended into the canopy. Pine seedlings were virtually absent.

The charred trunks of the pines indicated a history of occasional fires, which are often set by arsonists during the dry season. On 8 April 1996, a fire swept through most of the plantation and smouldered for several days ( $P$. Comeau, pers. comm.). Examination nearly two months later (2 June 1996) revealed that in most pines, the needles were brown in the lower half of the canopy and green in the upper half, while the trunks were blackened; there was no evidence of mortality. In contrast, most of the understorey vegetation was top-killed by the fire. The palm and dicot shrubs were leafless, with bare, blackened stems; the sedges were resprouting and had already attained a height of $1 \mathrm{~m}$ in some areas. We did not examine the larger dicot trees in the ravines. By August and September 1996, the sedges had once again attained a height of 1-2 m, and a variety of herbaceous plants were flowering in the understorey.

\section{Native forest}

The native broad-leaved forest, located just east of the pine plantation, comprised naturally regenerated secondary forest and a small, abandoned cocoa Theobroma cacao estate. The land had been protected as a watershed since ownership was transferred to the Mount Saint Benedict monastery in 1912; thus, the age of the secondary forest was at least 83 years (G. Ramsawak, pers. comm.).

The margin of the native forest adjacent to the pine forest was characterized by brush that had been frequently cleared as a firebreak. Elsewhere the height of the forest varied from 15 to $41+\mathrm{m}$, and was generally tallest along the course of a small stream that flowed through the valley but was reduced to a few small pools during the dry season. Away from the stream the forest included a few patches of shorter $(15-20 \mathrm{~m})$, relatively even-aged stands, with a few emergent older trees; charred stumps and multiple-stemmed trees indicated a previous history of fire and subsequent regeneration. Epiphytic plants were conspicuous at all strata of the forest. The undergrowth of the forest was relatively sparse. A few non-native (mostly naturalized) tree species were interspersed among the native tree species, but comprised a small percentage of the trees present. These 
included cocoa, breadfruit Artocarpus altilis, yellow poui Tabebuia serratifolia and mountain immortelle Erythrina micropteryx.

Beard (1946) described the dominant plant species and structure of the native forest at this elevation, which spans the transition from "deciduous seasonal forest" (lower elevations) to "lower montane rain forest" (upper elevations). The climate, soils, fauna and flora of the native forest at Mount Saint Benedict are similar to that described for the Arima Valley (Beebe 1952), located $12 \mathrm{~km}$ to the east of Mount Saint Benedict.

\section{Methods}

\section{Vegetation sampling}

We measured several parameters of the vegetation structure in each forest using the point-centered quarter method (e.g. Barbour et al. 1980). Points were randomly selected along narrow trails and the distance $(\mathrm{m})$ to the nearest tree with a diameter at breast height (DBH) greater than $4.0 \mathrm{~cm}$ within each of four $90^{\circ}$ sectors was measured to calculate density (stems $/ \mathrm{ha}$ ). The circumference $(\mathrm{m})$ of each tree at breast height $(\mathrm{CBH})$ was measured and later transformed into DBH. De: sity and mean DBH were used to calculate basal area $\left(\mathrm{m}^{2} / \mathrm{ha}\right)$. The height $(\mathrm{m})$ of each tree was measured with a clinometer using a percentage scale. The percentage canopy cover was measured at randomly selected points with a concave spherical densiometer (Lemmon 1957). We did not attempt to quantify the abundance and size classes of different plant species (except for Caribbean pine) within either forest.

\section{Bird sampling}

To sample bird abundance, we used the fixed-radius point count method (Hutto et al. 1986), which is currently a widely used method for censusing birds in both the understorey and canopy of forest habitats (e.g. Ralph et al. 1995). Although a variety of radii and time periods have been employed for point counts (e.g. Ralph et al. 1995), we used the proposed standards for censusing landbirds in the Caribbean (Wunderle 1994). From 15 October 1995 to 22 September 1996, we conducted 100 point counts in each habitat (pine forest and native forest). At each point we counted all birds (including unidentified individuals) seen or heard within a distance of $25 \mathrm{~m}$ for a period of 10 minutes; birds seen or heard flying above the canopy were excluded. Most birds seen were identified by their characteristic vocalizations or plumage characters (ffrench 1991), with which we were intimately acquainted from more than 2 years of experience in Trinidad.

To reduce time-of-day and seasonal biases in our comparisons between the two forests, we conducted 10 counts simultaneously in each habitat, with one observer in each habitat, during one morning (between o7hoo and 1ohoo) in each month of the year (except March and May 1996); the intervals between censuses (hereafter a census refers to a set of 10 counts conducted during a single morning) ranged from 25 to 58 days. F.E.H. conducted six censuses in the pine forest and four in the native forest; I. S. Samad conducted four in the pine forest and six in the native forest. All point counts were conducted along or near narrow trails 
with closed canopies within mature forest in each habitat and were at least $50 \mathrm{~m}$ from the edge of the habitat. No point counts were conducted during periods of rainfall or strong wind. In the pine plantation, the counts were conducted primarily along the central ridge or adjacent slopes where pines were essentially the only trees present; the ravines were avoided because broad-leaved native trees and shrubs were often present. In the native forest, the counts were conducted on the floor and slopes of a valley and along a ridge. Successive point counts were separated by a minimum distance of $100 \mathrm{~m}$, with no counts repeated at any point during a given census. We did not attempt to repeat counts at the same points during each census.

To facilitate comparisons of the ecological characteristics of the two bird communities, we placed each species into one of three habitat categories, five diet categories and three foraging strata categories, respectively. Placement into habitat categories was based on the habitat in which we have most frequently encountered each species in various parts of Trinidad: (1) forest interior, (2) forest edge, or (3) generalist, occurring in both the edge and interior of forests. Placement into diet categories was based on the primary constituents of each species's diet according to ffrench (1991): (1) frugivores, feeding on fruit or seeds; (2) nectarivores, feeding on the nectar of flowers; (3) insectivores, feeding on arthropods; (4) carnivores, feeding on vertebrates; and (5) omnivores, feeding roughly equally on plant and animal matter. Placement into foraging strata categories was based on our observations for each species in various parts of Trinidad: (1) air above forest; (2) canopy (upper two-thirds) of forest; and (3) understorey (lower one-third) of forest. The taxonomy and sequence of species follow the American Ornithologists' Union (1983) and ffrench (1991).

\section{Statistical analyses}

The statistical tests used include the Bartlett's test ( $F$ statistic), chi-square test ( $\chi^{2}$ statistic), Kruskal-Wallis test ( $H$ statistic) and Mann-Whitney $U$-test ( $z$ statistic); these tests and their assumptions are described by Zar (1984). The Shannon index of diversity $\left(H^{\prime}\right)$ was calculated as a measure of species diversity in each habitat and compared between the two habitats with the $t$ statistic ( $\operatorname{Zar} 1984$ ). Although censuses were repeated at different times of the year, they were conducted simultaneously with the same number of counts in each habitat during each census; thus, data pooled for the entire study period may be used for comparisons regardless of any seasonal variation that may have occurred. Because the counts were conducted simultaneously in each habitat, they may be treated as paired observations in analyses comparing the two habitats; however, we chose to compare counts as independent observations for all analyses because they were conducted at slightly different times (up to 30 minutes apart, but usually $<15$ minutes apart) and by different observers. All tests were computed with Statistix 3.1 software (Anon. 1990), with $\alpha=0.05$.

\section{Results}

\section{Vegetation structure}

The density of tree stems in the pine forest $(1,2.95$ stems/ha; exclusively $P$. caribaea along the trails surveyed) did not differ significantly from that of the native forest 
Table 1. Measurements for selected variables of vegetation structure in exotic pine forest (only Pinus caribaea along trails sampled) and native broad-leaved forest at Mount Saint Benedict, Trinidad

\begin{tabular}{lccccc}
\hline Variable & $\bar{x}$ & S.D. & Min & Max & $n$ \\
\hline Diameter at breast height $(\mathrm{m})^{a}$ & & & & & \\
$\quad$ Pine forest & 0.22 & 0.07 & 0.10 & 0.38 & 52 \\
$\quad$ Native forest & 0.28 & 0.26 & 0.04 & 1.23 & 52 \\
$\quad$ & & & & & 52 \\
Tree height $(\mathrm{m})^{b}$ & 14.2 & 2.86 & 7.1 & 21.1 & 52 \\
$\quad$ Pine forest & 14.9 & 9.23 & 2.9 & 41.0 & \\
$\quad$ Native forest & & & & & 52 \\
Canopy cover $(\%)$ & 91.1 & 8.98 & 57 & 100 & 52 \\
$\quad$ Pine forest & 98.9 & 3.22 & 79 & 100 & 52 \\
$\quad$ Native forest & & & & &
\end{tabular}

Means: $=0.13, P=0.89$; variability: $F=14.67, P<0.001$.

"Means: $==1.03, P=0.30$; variability: $F=10.41, P<0.001$.

Means: $=5.98, P<0.001$; variability: $F=5.36, P<0.001$

$(1,172 /$ ha; $z=0.97, P=0.33)$. The basal area of the native forest $\left(73.8 \mathrm{~m}^{2} / \mathrm{ha}\right)$ was greater than that of the pine forest $\left(50.9 \mathrm{~m}^{2} / \mathrm{ha}\right)$, but no statistical tests could be computed with the calculations used. The trees of the two forests did not differ significantly in mean DBH or tree height, but both of these measurements were significantly more variable in the native forest (Table 1 ). Percentage canopy cover was significantly greater and less variable in the native forest (Table 1 ).

\section{Potential bird sampling bias}

Because visibility is generally greater in the narrow-leaved pine forests than in the broad-leaved native forest, birds may be easier to detect and identify in the pine forest. Proportionately fewer individuals were positively identified (to species) in the native broad-leaved forest than in the pine forest $\left(\chi^{2}=3.83, d f=1\right.$, $P=0.05)$, but the difference was very small ( $92.0 \%$ of 689 detections and $94.9 \%$ of 623 detections, respectively).

Variation among observers in the detectability and identification of birds may cause bias. The proportion of identified birds did not vary significantly between observers in the pine forest (F.E.H., 93.8\%; I.S., 97.6\%; $\chi^{2}=2.97, d f=1, P=0.08$ ), but in the native forest I.S. identified proportionately more birds $(94.9 \%)$ than F.E.H. $\left(88.8 \% ; \chi^{2}=7.87, d f=1, P=0.005\right)$, with a difference of $6.1 \%$.

Time-of-day variation in the detectability of birds is another potential source of bias. When the data from all censuses were pooled, there was significant time-of-day variation in the number of individual birds detected per count in the native forest, but no significant variation in the pine forest or in the number of species identified per count in either habitat (Table 2).

Because birds may easily travel between points and be counted more than once, the distance between point counts may affect the independence of the data. The mean number of individual birds detected per point count did not vary between counts spaced apart at intervals of $100 \mathrm{~m}(n=100)$ or $200 \mathrm{~m}(n=50)$ in either the pine forest (for $100 \mathrm{~m}, \bar{x}=6.23$, S.D. $=4.41$; for $200 \mathrm{~m}, \bar{x}=5.96$, S.D. $=3.53$; $z=0.05, P=0.96$ ) or the native forest (for $100 \mathrm{~m}, \bar{x}=6.90, S . D .=4.60$; for $200 \mathrm{~m}, \bar{x}=$ 7.44 , S.D. $=4.70 ; z=0.86, P=0.39$ ). 
Table 2. Time-of-day variation in the abundance of birds for all censuses combined in exotic pine forest and native broad-leaved forest at Mount Saint Benedict, Trinidad

\begin{tabular}{|c|c|c|c|c|c|c|c|c|}
\hline \multirow[t]{2}{*}{ Time of day } & \multicolumn{4}{|c|}{ Pine forest } & \multicolumn{4}{|c|}{ Native forest } \\
\hline & $\bar{x}$ & S.D. & $n$ & Range & $\bar{x}$ & S.D. & $n$ & Range \\
\hline \multicolumn{9}{|c|}{ Number of birds per count" } \\
\hline $07-00-08-00$ & 6.77 & 5.17 & 30 & $0-28$ & 7.97 & 4.24 & 34 & $1-23$ \\
\hline $08-00-09-00$ & 6.00 & 3.94 & 43 & $0-17$ & 5.77 & 4.73 & 39 & $0-23$ \\
\hline $09-00-10-00$ & 6.00 & $4 \cdot 32$ & 27 & $0-15$ & 7.15 & 4.52 & 27 & $2-19$ \\
\hline \multicolumn{9}{|c|}{ Number of species per count ${ }^{h}$} \\
\hline $07-00-08-00$ & 3.63 & 1.77 & 30 & $0-8$ & 4.68 & 2.36 & 34 & $1-13$ \\
\hline $08-00-09-00$ & 3.28 & 1.76 & 43 & $0-7$ & 3.64 & 2.22 & 39 & $0-8$ \\
\hline $09-00-10-00$ & 3.15 & 2.08 & 27 & $0-8$ & 4.89 & 3.12 & 27 & $1-14$ \\
\hline
\end{tabular}

"For pine forest: $H=0.42, P=0.81$; for native forest: $H=7.14, P=0.03$.

"For pine forest: $H=0.69, P=0.71$; for native forest: $H=4.06, P=0.13$.

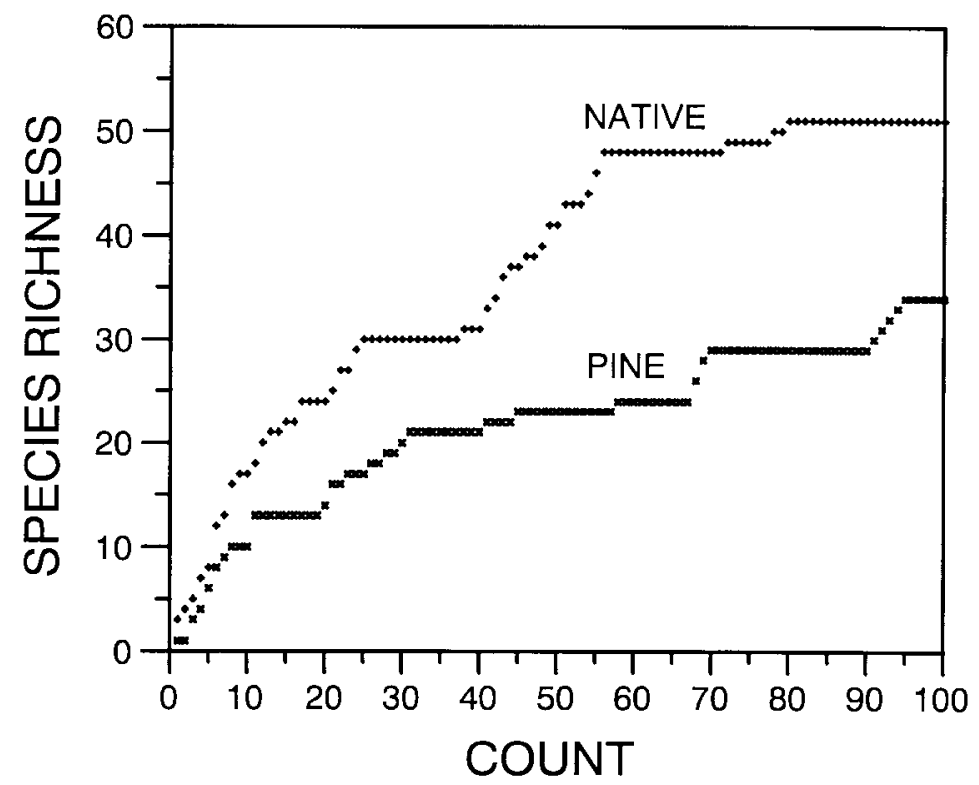

Figure 2. Species-accumulation curves for birds identified in a Caribbean pine plantation and native broad-leaved forest at Mount Saint Benedict, Trinidad.

A comparison of bird species richness between two habitats requires adequate sampling. The species-accumulation curve for the native forest essentially reached an asymptote, with no new species detected after 80 counts; in contrast, new species continued to be added to the pine forest throughout the study period, including five new species during counts 90-95 (Figure 2).

\section{Avifaunal comparisons}

Species richness was significantly higher in the native forest: of 66 species recorded during the counts, $51(77.3 \%)$ were found in the native forest whereas 
Table 3. Statistical comparisons for frequency of counts detected ( $\chi^{2}$ statistic) and mean number of birds per count (Mann-Whitney $U$-test; $z$ statistic) for each species in exotic pine forest and native broad-leaved forest at Mount Saint Benedict, Trinidad. Statistical tests are provided only for species detected in six or more counts $(n=200)$, which is the minimum required to detect statistical differences with the chi-square test. Habitat: $E$, forest edge; $F$, forest interior; $G$, forest interior and edge. Diet: C, carnivores; F, frugivores (fruit/seeds); I, insectivores; N, nectarivores; O, omnivores. Strata: A, aerial; $C$, canopy; $U$, understorey

\begin{tabular}{|c|c|c|c|c|c|c|c|c|c|}
\hline \multirow[t]{2}{*}{ Species } & \multicolumn{3}{|c|}{ Frequency } & \multicolumn{3}{|c|}{ Mean number/count } & \multirow[b]{2}{*}{ Habitat } & \multirow[b]{2}{*}{ Diet } & \multirow[b]{2}{*}{ Strata } \\
\hline & Pine & Native & $\chi^{2}$ & Pine & Native & $z$ & & & \\
\hline $\begin{array}{l}\text { Little Tinamou } \\
\text { Crypturellus soui }\end{array}$ & - & 0.03 & - & - & 0.3 & - & $F$ & $\mathrm{O}$ & $\mathrm{U}$ \\
\hline $\begin{array}{l}\text { Black Vulture } \\
\text { Coragyps atratus }\end{array}$ & 0.09 & - & $7 \cdot 45^{b}$ & 0.16 & - & 1.10 & G & C & A \\
\hline $\begin{array}{l}\text { Turkey Vulture } \\
\text { Cathartes anra }\end{array}$ & 0.05 & - & - & 0.06 & - & - & G & C & A \\
\hline $\begin{array}{l}\text { White Hawk } \\
\text { Leucopternis albicollis }\end{array}$ & 0.01 & - & - & 0.01 & - & - & $\mathrm{F}$ & C & A \\
\hline $\begin{array}{l}\text { Yellow-headed Caracara } \\
\text { Miliago chimachima }\end{array}$ & 0.01 & - & - & 0.01 & - & - & $E$ & C & A \\
\hline $\begin{array}{l}\text { Scaled Pigeon } \\
\text { Columba speciosa }\end{array}$ & - & 0.04 & - & - & 0.04 & - & $\mathrm{F}$ & $\mathrm{F}$ & C \\
\hline $\begin{array}{l}\text { Ruddy Ground-Dove } \\
\text { Columbina talpacoti }\end{array}$ & 0.06 & - & $4 \cdot 30^{6}$ & 0.39 & - & 0.73 & $\mathrm{E}$ & $\mathrm{F}$ & U \\
\hline $\begin{array}{l}\text { White-tipped Dove } \\
\text { Leptotila ierreanxi }\end{array}$ & 0.01 & - & - & 0.01 & - & - & $\mathrm{G}$ & $\mathrm{F}$ & $\mathrm{U}$ \\
\hline $\begin{array}{l}\text { Gray-fronted Dove } \\
\text { Leptotila rufaxilla }\end{array}$ & - & 0.5 & - & - & 0.06 & - & $\mathrm{F}$ & $\mathrm{F}$ & $\mathrm{U}$ \\
\hline $\begin{array}{l}\text { Orange-winged } \\
\text { Parrot Amazona amazonica } \\
\text { Squirrel Cuckoo }\end{array}$ & - & 0.07 & $5 \cdot 33^{21}$ & - & 0.29 & 0.85 & $\mathrm{~F}$ & $\mathrm{~F}$ & $\mathrm{C}$ \\
\hline Piaya cayana & - & 0.05 & - & - & 0.05 & - & $\mathrm{F}$ & I & $\mathrm{C}$ \\
\hline $\begin{array}{l}\text { Short-tailed Swift } \\
\text { Chaetura brachyura }\end{array}$ & 0.01 & - & - & 0.01 & - & - & G & I & A \\
\hline $\begin{array}{l}\text { Rufous-breasted Hermit } \\
\text { Glaucis hirsuta }\end{array}$ & - & 0.09 & $7 \cdot 45^{b}$ & - & 0.13 & 1.10 & G & $\mathrm{N}$ & $\mathrm{U}$ \\
\hline $\begin{array}{l}\text { Green Hermit } \\
\text { Plathlormis guy }\end{array}$ & - & 0.11 & $9.62^{k}$ & - & 0.11 & 1.34 & $\mathrm{~F}$ & $\mathrm{~N}$ & $\mathrm{U}$ \\
\hline $\begin{array}{l}\text { Little Hermit } \\
\text { Placthornis longuenureus }\end{array}$ & - & 0.04 & - & - & 0.07 & - & $\mathrm{F}$ & $\mathrm{N}$ & $\mathrm{U}$ \\
\hline $\begin{array}{l}\text { Black-throated Mango } \\
\text { Anthracothorax nigricollis }\end{array}$ & 0.02 & 0.02 & - & 0.02 & 0.03 & - & $E$ & $N$ & $\mathrm{C}$ \\
\hline $\begin{array}{l}\text { Blue-chinned Sapphire } \\
\text { Chlorestes notatus }\end{array}$ & - & 0.01 & - & - & 0.02 & - & $\mathrm{F}$ & $\mathrm{N}$ & C \\
\hline $\begin{array}{l}\text { Copper-rumped } \\
\text { Hummingbird } \\
\text { Amazilia tobact }\end{array}$ & $0.7^{\circ}$ & 0.12 & $67.16^{i}$ & 0.99 & 0.19 & $6.88^{i}$ & $E$ & $N$ & $\mathrm{U}$ \\
\hline $\begin{array}{l}\text { White-tailed Trogon } \\
\text { Trogon ividis }\end{array}$ & - & 0.04 & - & - & 0.04 & - & $\mathrm{F}$ & $\mathrm{O}$ & $\mathrm{C}$ \\
\hline $\begin{array}{l}\text { Collared Trogon } \\
\text { Trogon collarts }\end{array}$ & - & 0.01 & - & - & 0.01 & - & $\mathrm{F}$ & $\mathrm{O}$ & $\mathrm{C}$ \\
\hline $\begin{array}{l}\text { Channel-billed Toucan } \\
\text { Ramphastos zitellinus }\end{array}$ & - & 0.01 & - & - & 0.01 & - & $F$ & $F$ & C \\
\hline
\end{tabular}


Table 3.- (contd.)

\begin{tabular}{|c|c|c|c|c|c|c|c|c|c|}
\hline \multirow[t]{2}{*}{ Species } & \multicolumn{3}{|c|}{ Frequency } & \multicolumn{3}{|c|}{ Mean number/count } & \multirow[b]{2}{*}{ Habitat } & \multirow[b]{2}{*}{ Diet } & \multirow[b]{2}{*}{ Strata } \\
\hline & Pine & Native & $\chi^{2}$ & Pine & Native & $z$ & & & \\
\hline $\begin{array}{l}\text { Red-rumped Woodpecker } \\
\text { Veniliornis kirkii }\end{array}$ & - & 0.01 & - & - & 0.02 & - & G & I & $\mathrm{C}$ \\
\hline $\begin{array}{l}\text { Lineated Woodpecker } \\
\text { Dryocopus lineatus }\end{array}$ & 0.01 & 0.03 & - & 0.01 & 0.03 & - & $G$ & I & C \\
\hline $\begin{array}{l}\text { Streaked Xenops } \\
\text { Xenops rutilans }\end{array}$ & - & 0.02 & - & - & 0.03 & - & $\mathrm{F}$ & I & C \\
\hline $\begin{array}{l}\text { Gray-throated Leaftosser } \\
\text { Sclerurus albigularis }\end{array}$ & - & 0.01 & - & - & 0.01 & - & $\mathrm{F}$ & I & $\mathrm{U}$ \\
\hline $\begin{array}{l}\text { Plain-brown Woodcreeper } \\
\text { Dendrocincla fuliginosa }\end{array}$ & - & 0.03 & - & - & 0.03 & - & $\mathrm{F}$ & I & $\mathrm{U}$ \\
\hline $\begin{array}{l}\text { Buff-throated Woodcreeper } \\
\text { Xiphorhynchus guttatus }\end{array}$ & - & 0.10 & $8.53^{i^{n}}$ & - & 0.10 & 1.22 & $\mathrm{~F}$ & I & $\mathrm{U}$ \\
\hline $\begin{array}{l}\text { Great Antshrike } \\
\text { Taraba mtajor }\end{array}$ & - & 0.01 & - & - & 0.01 & - & $F$ & I & $\mathrm{U}$ \\
\hline $\begin{array}{l}\text { White-flanked Antwren } \\
\text { Myrmotherula axillaris }\end{array}$ & - & 0.16 & $15.29^{\circ}$ & - & 0.23 & $1.95^{x}$ & $\mathrm{~F}$ & I & $\mathrm{U}$ \\
\hline $\begin{array}{l}\text { White-bellied Antbird } \\
\text { Mymeciza longipes }\end{array}$ & - & 0.03 & - & - & 0.03 & - & $F$ & I & $\mathrm{U}$ \\
\hline $\begin{array}{l}\text { Southern Beardless- } \\
\text { Tyrannulet Camptostonta } \\
\text { obsoletum }\end{array}$ & 0.17 & 0.14 & 0.15 & 0.23 & .16 & 0.41 & $F$ & 1 & $\mathrm{C}$ \\
\hline $\begin{array}{l}\text { Ochre-bellied Flycatcher } \\
\text { Mionectes oleaginea }\end{array}$ & - & 0.06 & $4 \cdot 30^{2} \mathrm{a}$ & - & 0.06 & 0.73 & $F$ & I & $\mathrm{U}$ \\
\hline $\begin{array}{l}\text { Yellow-breasted Flycatcher } \\
\text { Tolntomyias flaviventris }\end{array}$ & 0.02 & 0.11 & $5.27^{n}$ & 0.03 & 0.13 & 1.09 & $\mathrm{~F}$ & I & $C$ \\
\hline $\begin{array}{l}\text { Euler's Flytcatcher } \\
\text { Lathrotriccus euleri }\end{array}$ & 0.02 & - & - & 0.02 & - & - & $F$ & I & C \\
\hline $\begin{array}{l}\text { Dusky-capped Flycatcher } \\
\text { Myiarchus tuberculifer }\end{array}$ & 0.01 & - & - & 0.01 & - & - & $\mathrm{E}$ & I & C \\
\hline $\begin{array}{l}\text { Brown-crested Flycatcher } \\
\text { Myiarchus tyrannulus }\end{array}$ & 0.01 & - & - & 0.01 & - & - & $E$ & I & C \\
\hline $\begin{array}{l}\text { Great Kiskadee } \\
\text { Pitangus sulphuratus }\end{array}$ & 0.06 & 0.20 & $7 \cdot 47^{b}$ & 0.06 & 0.25 & 1.75 & $E$ & I & $\mathrm{C}$ \\
\hline $\begin{array}{l}\text { Boat-billed Flycatcher } \\
\text { Megarynchus pitangua }\end{array}$ & - & 0.03 & - & - & 0.04 & - & G & I & C \\
\hline $\begin{array}{l}\text { White-winged Becard } \\
\text { Pachyramphus polychopterus }\end{array}$ & 0.01 & - & - & 0.01 & - & - & $F$ & I & C \\
\hline $\begin{array}{l}\text { White-bearded Manakin } \\
\text { Manacus manacus }\end{array}$ & - & 0.11 & $9.62^{k^{i}}$ & - & 0.14 & 1.34 & $\mathrm{~F}$ & $\mathrm{O}$ & $\mathrm{U}$ \\
\hline $\begin{array}{l}\text { Golden-headed Manakin } \\
\text { Pipra erythrocephala }\end{array}$ & 0.01 & 0.22 & $19.65^{i}$ & 0.01 & 0.41 & $2.58^{h}$ & $\mathrm{~F}$ & $\mathrm{O}$ & C \\
\hline $\begin{array}{l}\text { Rufous-breasted Wren } \\
\text { Thryothorus rutilus }\end{array}$ & 0.08 & 0.14 & 1.28 & 0.08 & 0.16 & 0.75 & $F$ & I & $\mathrm{U}$ \\
\hline $\begin{array}{l}\text { House Wren } \\
\text { Troglodytes aedon }\end{array}$ & 0.15 & - & $14.13^{c}$ & 0.17 & - & 1.83 & $\mathrm{E}$ & I & $\mathrm{U}$ \\
\hline $\begin{array}{l}\text { Long-billed Gnatwren } \\
\text { Ramphocaenus melanurus }\end{array}$ & 0.01 & 0.20 & $17.24^{\circ}$ & 0.01 & 0.25 & $2.33^{\prime \prime}$ & $\mathrm{F}$ & I & C \\
\hline $\begin{array}{l}\text { Cocoa Thrush } \\
\text { Turdus fumigatus }\end{array}$ & - & 0.08 & $6.38^{a}$ & - & 0.08 & 0.98 & $\mathrm{~F}$ & $\mathrm{O}$ & $\mathrm{U}$ \\
\hline
\end{tabular}


Table 3.-(contd.)

\begin{tabular}{|c|c|c|c|c|c|c|c|c|c|}
\hline \multirow[t]{2}{*}{ Species } & \multicolumn{3}{|c|}{ Frequency } & \multicolumn{3}{|c|}{ Mean number/count } & \multirow[b]{2}{*}{ Habitat } & \multirow[b]{2}{*}{ Diet } & \multirow[b]{2}{*}{ Strate } \\
\hline & Pine & Native & $\chi^{2}$ & Pine & Native & $z$ & & & \\
\hline $\begin{array}{l}\text { Bare-eyed Thrush } \\
\text { Turdus nudigenis }\end{array}$ & 0.03 & 0.15 & $7 \cdot 39^{h}$ & 0.03 & 0.15 & 1.47 & $E$ & $\mathrm{O}$ & $\mathrm{U}$ \\
\hline $\begin{array}{l}\text { Red-eyed Vireo } \\
\text { Vireo oliviaceus }\end{array}$ & - & 0.03 & - & - & 0.03 & - & $\mathrm{F}$ & I & C \\
\hline $\begin{array}{l}\text { Golden-fronted Greenlet } \\
\text { Hylophilus aurantiifrons }\end{array}$ & - & 0.41 & $49.09^{c}$ & - & 0.67 & $5.01^{\circ}$ & $\mathrm{F}$ & I & C \\
\hline $\begin{array}{l}\text { Rufous-browed } \\
\text { Peppershrike }\end{array}$ & & & & & & & & & \\
\hline Cyclarhis gujanensis & - & 0.05 & - & - & 0.05 & - & $\mathrm{F}$ & I & $\mathrm{C}$ \\
\hline $\begin{array}{l}\text { Tropical Parula } \\
\text { Parula pitiayumi }\end{array}$ & 0.17 & 0.25 & 1.48 & 0.19 & 0.28 & 1.00 & $\mathrm{~F}$ & I & C \\
\hline $\begin{array}{l}\text { Golden-crowned Warbler } \\
\text { Basileuterus culicivorus }\end{array}$ & 0.01 & 0.11 & $7 \cdot 18^{b}$ & 0.01 & 0.18 & 1.23 & $\mathrm{~F}$ & I & $\mathrm{U}$ \\
\hline $\begin{array}{l}\text { Bananaquit } \\
\text { Coereba flazeola }\end{array}$ & 0.60 & 0.32 & $15.7^{8^{i}}$ & 1.00 & 0.46 & $3.88^{i 2}$ & G & $\mathrm{N}$ & C \\
\hline $\begin{array}{l}\text { Turquoise Tanager } \\
\text { Tangara mexicanta }\end{array}$ & 0.25 & 0.03 & $18.31^{\circ}$ & 0.62 & 0.09 & $2.67^{1}$ & $\mathrm{E}$ & $\mathrm{O}$ & C \\
\hline $\begin{array}{l}\text { Bay-headed Tanager } \\
\text { Tangara gyrola }\end{array}$ & 0.03 & 0.03 & 0.00 & 0.03 & 0.05 & 0.01 & $\mathrm{~F}$ & $\mathrm{O}$ & C \\
\hline $\begin{array}{l}\text { Purple Honeycreeper } \\
\text { Cyanerpes caeruleus }\end{array}$ & 0.01 & - & - & 0.01 & - & - & $\mathrm{F}$ & $\mathrm{O}$ & C \\
\hline $\begin{array}{l}\text { Red-legged Honeycreeper } \\
\text { Cyanerpes cyaneus }\end{array}$ & 0.01 & - & - & 0.01 & - & - & $\mathrm{F}$ & $\mathrm{O}$ & C \\
\hline $\begin{array}{l}\text { Violaceous Euphonia } \\
\text { Euphonia violacea }\end{array}$ & - & 0.03 & - & - & 0.04 & - & $\mathrm{E}$ & $F$ & C \\
\hline $\begin{array}{l}\text { Blue-grey Tanager } \\
\text { Thraupis episcopus }\end{array}$ & 0.07 & 0.11 & 0.55 & 0.09 & 0.30 & 0.54 & $G$ & $\mathrm{O}$ & C \\
\hline $\begin{array}{l}\text { Palm Tanager } \\
\text { Thraupis palmarum }\end{array}$ & 0.23 & 0.30 & 0.92 & 0.42 & 0.63 & 0.90 & $\mathrm{G}$ & $\mathrm{O}$ & C \\
\hline $\begin{array}{l}\text { White-shouldered Tanager } \\
\text { Tachyphonus luctuosus }\end{array}$ & - & 0.01 & - & - & 0.01 & - & $\mathrm{F}$ & $\mathrm{O}$ & C \\
\hline $\begin{array}{l}\text { White-lined Tanager } \\
\text { Tachyphonus rufus }\end{array}$ & 0.08 & 0.06 & 0.08 & 0.14 & 0.07 & 0.26 & $\mathrm{~F}$ & 0 & C \\
\hline $\begin{array}{l}\text { Red-crowned Ant-Tanager } \\
\text { Habia rubica }\end{array}$ & - & 0.04 & - & - & 0.04 & - & $\mathrm{F}$ & $\mathrm{O}$ & $\mathrm{U}$ \\
\hline $\begin{array}{l}\text { Silver-beaked Tanager } \\
\text { Ramphocelus carbo }\end{array}$ & - & 0.02 & - & - & 0.02 & - & $\mathrm{E}$ & $\mathrm{O}$ & $\mathrm{U}$ \\
\hline $\begin{array}{l}\text { Blue-black Grassquit } \\
\text { Volatinia jacarina }\end{array}$ & 0.43 & - & $52.26^{\circ}$ & 1.03 & - & $5.25^{\circ}$ & $\mathrm{E}$ & $\mathrm{O}$ & $U$ \\
\hline $\begin{array}{l}\text { Sooty Grassquit } \\
\text { Tiaris fuliginosa }\end{array}$ & 0.02 & 0.01 & - & 0.02 & 0.01 & - & $\mathrm{F}$ & $\mathrm{F}$ & $\mathrm{U}$ \\
\hline $\begin{array}{l}\text { Crested Oropendola } \\
\text { Psarocolius decumanus }\end{array}$ & - & 0.01 & - & - & 0.01 & - & $E$ & $\mathrm{O}$ & C \\
\hline
\end{tabular}


only $34(51.5 \%)$ were found in the pine forest $\left(\chi^{2}=8.46, d f=1, P=0.001\right.$; Figure 2, Table 3). Species diversity was significantly higher in the native forest $\left(H^{\prime}=1.47\right)$ than in the pine forest $\left(H^{\prime}=1.10 ; t=14.62, d f=1174, P<0.001\right)$. The number of individual birds detected per count was slightly - but not significantly - higher in the native forest $(\bar{x}=6.90$, S.D. $=4.60)$ than in the pine forest $(\bar{x}=6.23$, S.D. $=4.41$; $z=1.03, P=0.30$ ). The number of species identified per count was significantly higher in the native forest $(\bar{x}=4.33$, S.D. $=2.57)$ than in the pine forest $(\bar{x}=3.45$, S.D. $=$ 1.84; $z=2.37, P=0.018$ ).

Many species of birds were detected too infrequently for statistical comparisons of abundance between the two habitats. Chi-square tests, based on the frequency of counts recorded for each species, revealed that seven species were significantly more common in the pine forest, 15 species were significantly more common in the native forest, and seven species were equally common in both habitats (Table 3). Mann-Whitney $U$-tests, based on the mean number of birds per count in each habitat, revealed that four species were significantly more common in the pine forest, four species were significantly more common in the native forest, and 21 species were equally common in both habitats (Table 3).

The number of individual birds detected per count differed significantly between months in the pine forest $(H=47.54, P<0.001)$, but not in the native forest ( $H=13.84, P=0.13$ ). The number of species identified per count differed significantly between months in the pine forest $(H=34.46, P<0.001)$, and nearly significantly in the native forest $(H=16.51, P=0.057)$. In the pine forest, the abundance of individual birds was greatest during the months of August and September, and lowest in April, with no marked decrease after the fire on 8 April; in the native forest, the abundance of birds was greatest in February and July, and lowest during November (Figure 3).

Kruskal-Wallis tests revealed significant monthly variation in abundance for five species in the pine forest and 1o species in the native forest, but the pattern for most species was erratic. Mann-Whitney $U$-tests revealed a significant difference in abundance between the dry and wet seasons only for the Bananaquit Coereba flaveola in the native forest (for dry season, $\bar{x}=0.75$ birds/ count; for wet season, $\bar{x}=0.27$ / count; $z=2.07, P=0.04$ ).

The general habitat characteristics of birds inhabiting the two forests differed significantly at both the individual $\left(\chi^{2}=365.8, d f=2, P<0.001\right)$ and species levels $\left(\chi^{2}=6.03, d f=2, P=0.049\right.$; Figure 4$)$. Birds characteristically inhabiting the forest interior were proportionately more common in the native forest; birds inhabiting forest edges or both the interior and edges of forests (i.e. habitat generalists) were proportionately more common in the pine forest (Figure 4 ).

The general dietary characteristics of birds inhabiting the two forests differed significantly at the individual level $\left(\chi^{2}=162.2, d f=4, P<0.001\right)$, but not at the species level $\left(\chi^{2}=6.63, d f=4, P=0.16\right.$; Figure 5). At the individual level, nectarivores were proportionately more common in the pine forest whereas insectivores were more common in the native forest (Figure 5). Carnivores were not recorded within the native forest (Figure 5), but were often observed soaring overhead.

The proportion of birds foraging at different vertical strata differed significantly between the two forests at both the individual $\left(\chi^{2}=74.09, d f=2\right.$, 

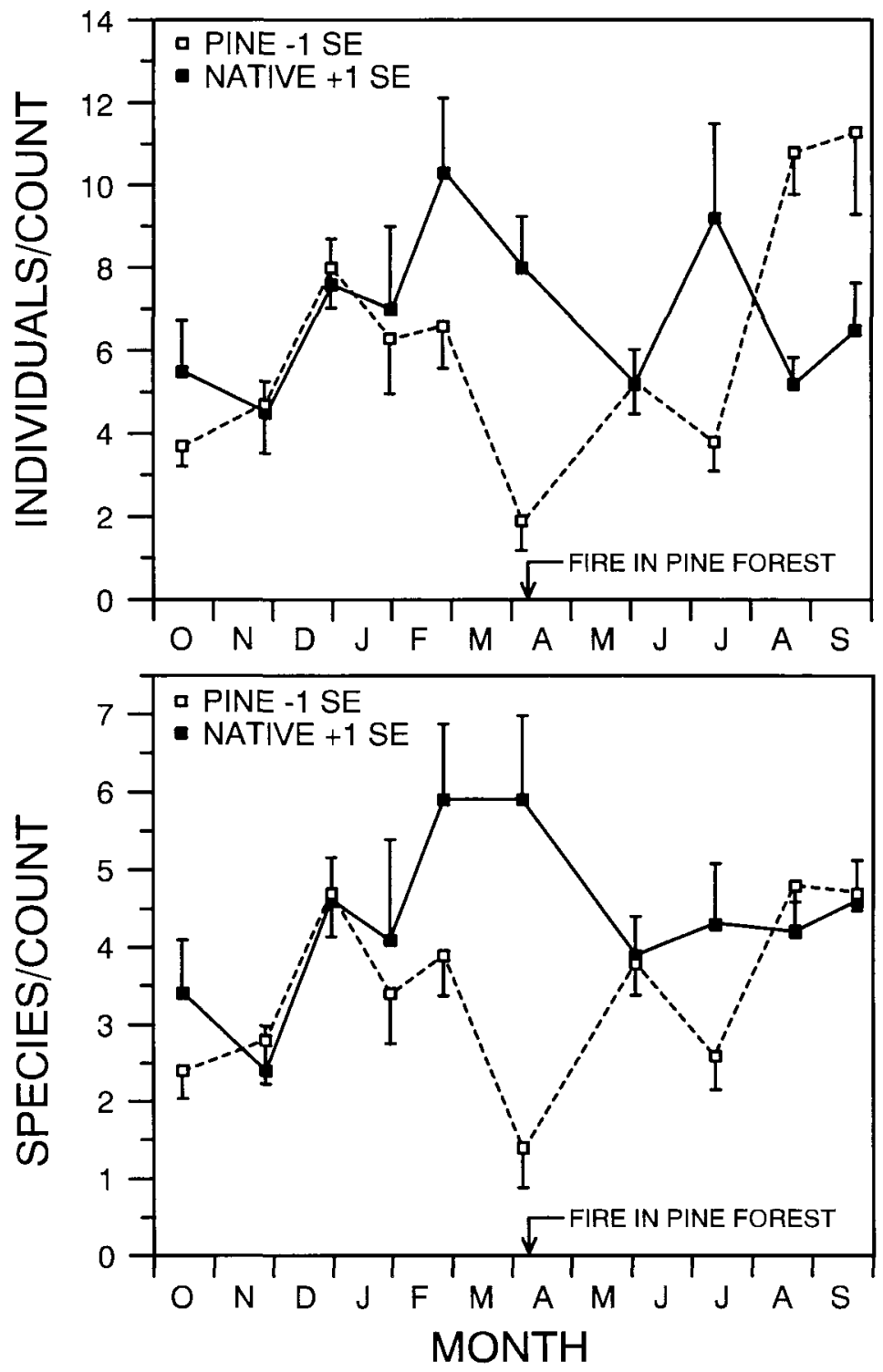

Figure 3. Seasonal variation (from October 1995 to September 1996) in the mean number of individual birds detected per count and mean number of species identified per count in a Caribbean pine plantation and native broad-leaved forest at Mount Saint Benedict, Trinidad.

$P<0.001)$ and species levels $\left(\chi^{2}=8.75, d f=2, P=0.01\right)$. At the individual level, more aerial and understorey foragers occurred in the pine forest; canopy foragers were more common in the native forest (Figure 6). At the species level, more aerial foragers occurred in the pine forest and more understorey foragers in the native forest (Figure 6). 

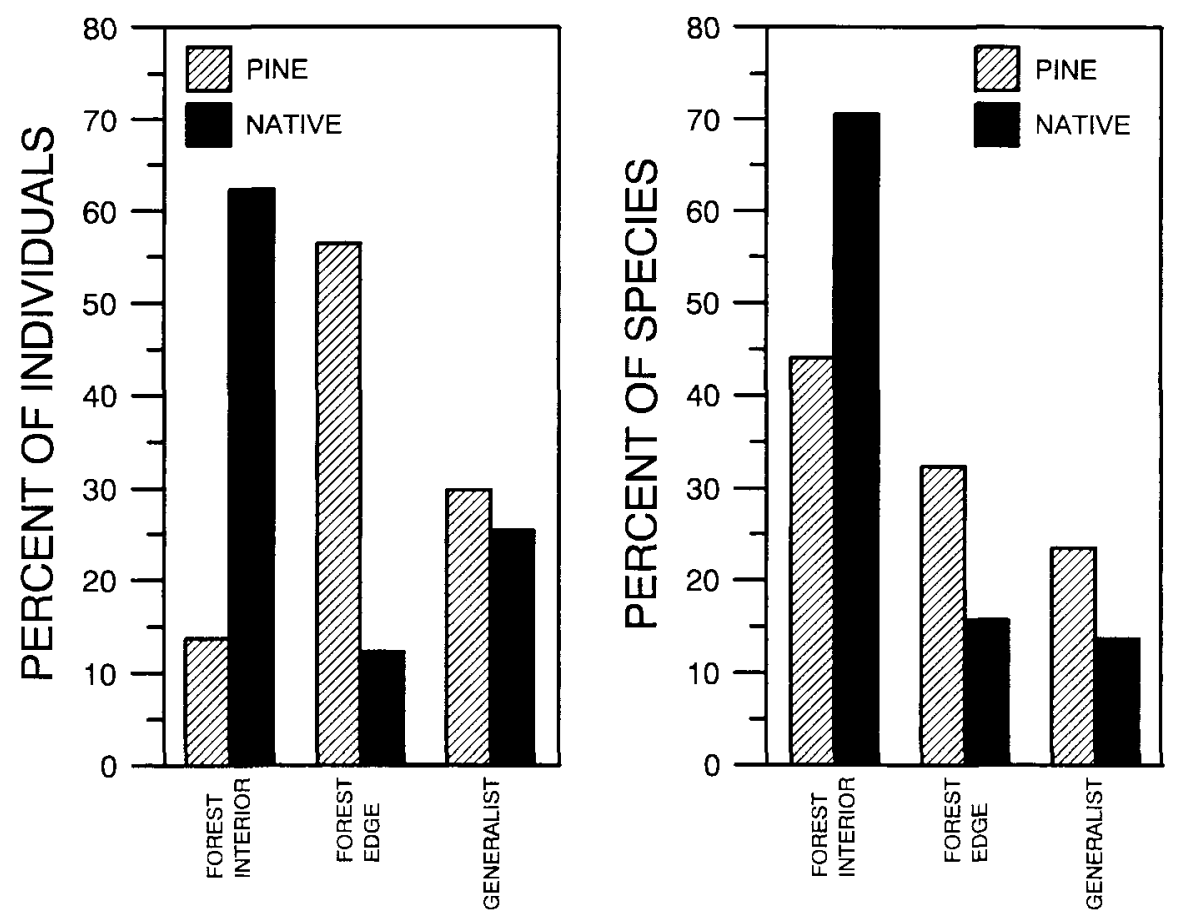

Figure 4. Percentage of individuals and species of birds characteristically occupying forest interior, forest edge or both (generalist) habitats in a Caribbean pine plantation and native broad-leaved forest at Mount Saint Benedict, Trinidad.

\section{Discussion}

\section{Vegetation structure}

Although the mean density, width and height of trees did not differ significantly between the two forests, the width and height of trees were considerably more variable in the native forest. These results indicate that the native forest, which comprises many tree species, is structurally more heterogeneous than the pine-dominated forest. The more consistent and uniform canopy cover and broader leaves of trees in the native forest reduce the amount of light penetrating to the forest floor, thus restricting the growth of understorey plants.

\section{Potential bird sampling bias}

We have attempted to analyse only a few of the potential sources of bird sampling bias (for studies of these and other potential sources of bias, see Ralph and Scott 1981, Verner 1985, Bibby et al. 1992, Ralph et al. 1995). Because the leaves are considerably broader in the native forest than in the pine forest, visibility was probably more restricted in the native forest; this may explain why proportionately fewer birds were identified in the native forest, though the difference was quite small (2.9\%). Birds may well have been detected less 

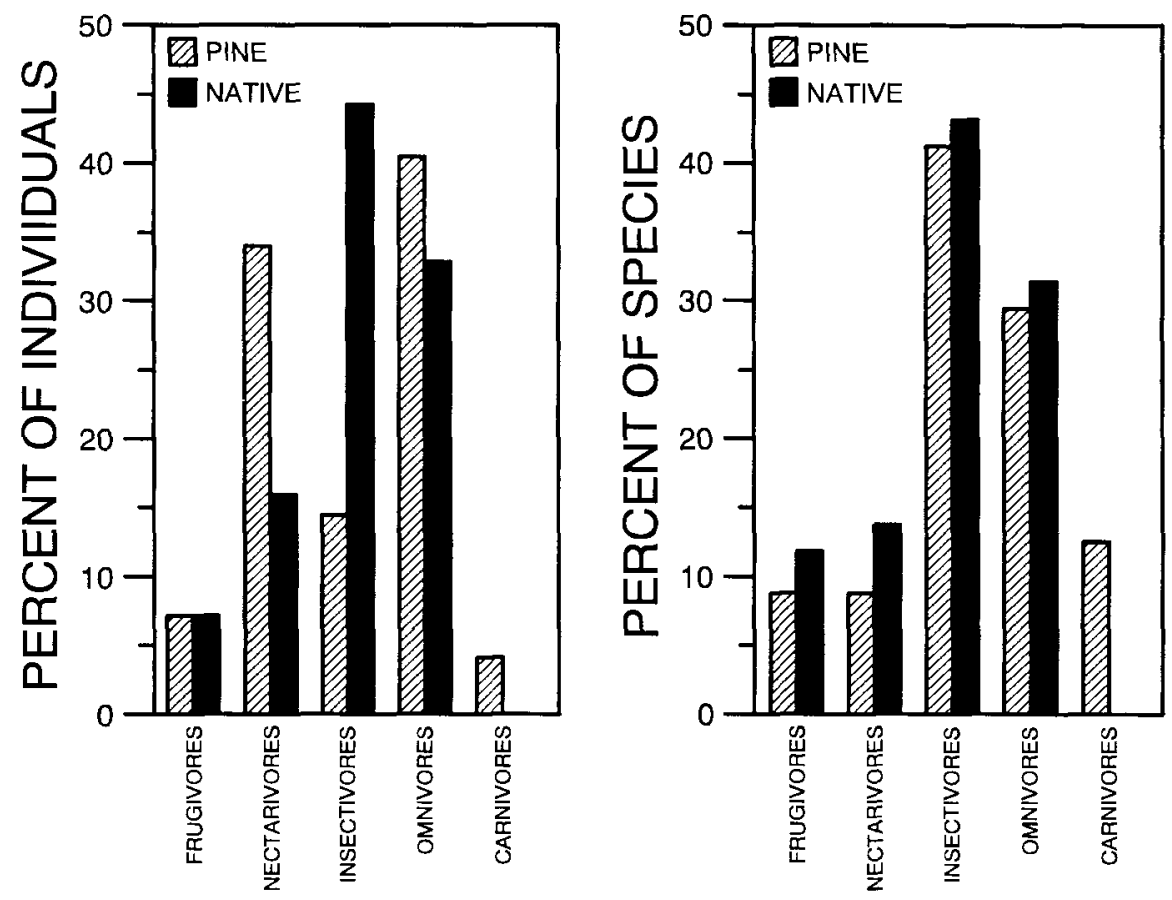

Figure 5. Percentage of individuals and species of birds belonging to various diet categories in a Caribbean pine plantation and native broad-leaved forest at Mount Saint Benedict, Trinidad.

frequently in the native broad-leaved forest, but this hypothesis cannot be tested. These potential sources of bias suggest that the birds were undersampled in the native forest in comparison with the pine forest. Variation between observers in the identification and detectability of birds is a long-recognized problem, even among experienced observers, and may complicate the interpretation of our results. However, neither observer conducted more than $60 \%$ of the counts in either habitat. Ideally an equal number of counts should have been conducted by each observer in each habitat, which would minimize observer bias. Because the counts in each habitat were conducted simultaneously, time-of-day variation in our pooled comparisons should be minimal.

The spacing of point counts only $100 \mathrm{~m}$ apart had no measurable effect on the independence of the data, but would probably affect the independence of the data for point counts of a larger (e.g. $50 \mathrm{~m}$ ) or unlimited radius. The species-accumulation curve for the native forest essentially reached an asymptote during the study period, suggesting that most of the species in the study area during the study period were recorded. However, the addition of new species to the pine forest throughout the study suggests that additional counts would have continued to accumulate more species. We suspect that many of the birds identified in the pine forest were transient visitors rather than breeding residents, which might explain why new species continued to be observed in the pine forest throughout the study. An additional 22 species of birds have been recorded 

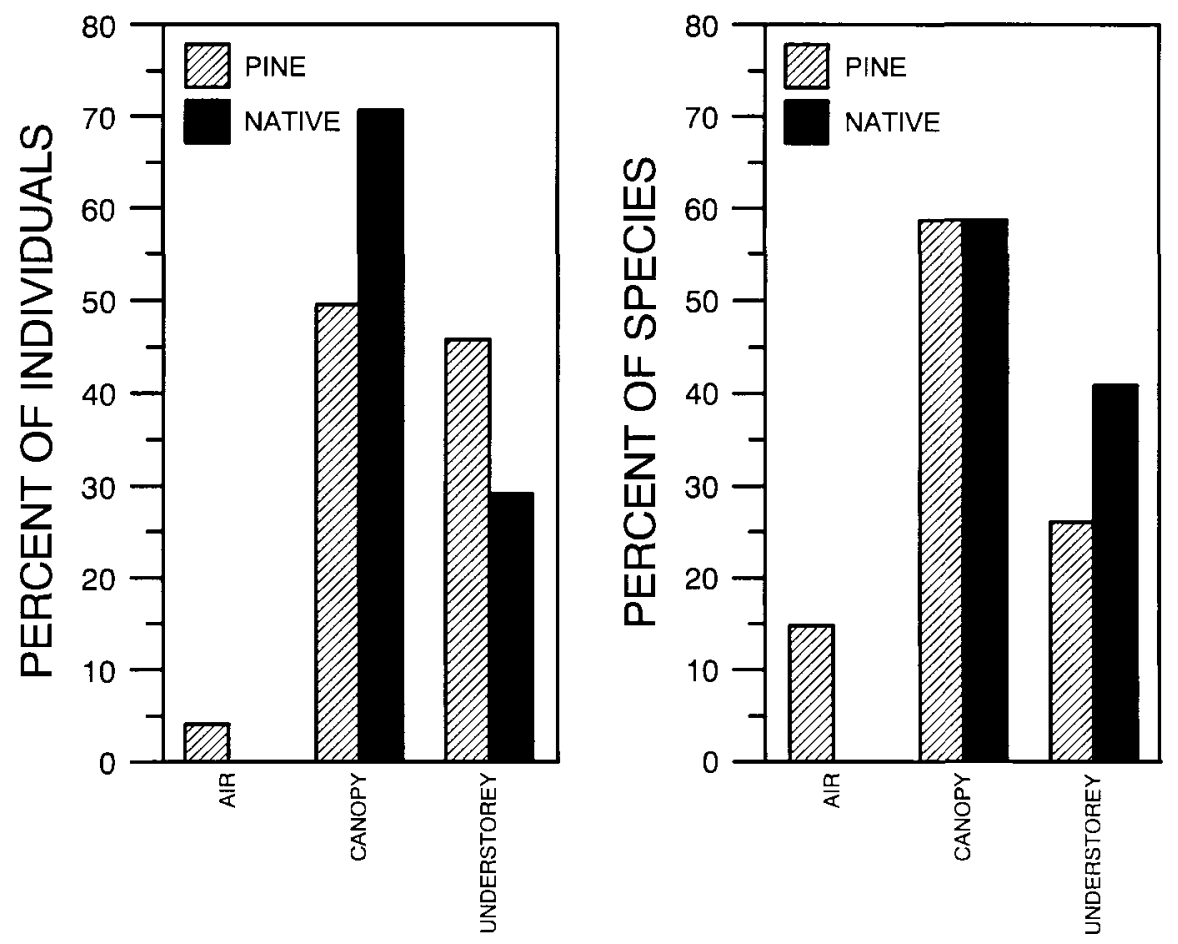

Figure 6. Percentage of individuals and species of birds foraging at different vertical strata in a Caribbean pine plantation and native broad-leaved forest at Mount Saint Benedict, Trinidad.

within the pine forest and 13 flying over, and an additional 32 species within the native forest and 15 flying over (G. Ramsawak, F.E.H. and I.S., unpublished); however, our data indicate that individuals of the additional species combined account for less than $1 \%$ of the population in each community, respectively. Because extensive sampling bias generally reduces the probability of finding significant differences between comparisons, the numerous significant comparisons documented in this study suggest that the various sources of bias were minor and did not invalidate our comparisons.

\section{Faunal comparisons}

The broad-leaved native forest provided a more heterogeneous habitat than the native forest, with more species of plants and more foliage per unit area, and presumably provided more flowers, fruits and arthropods as food resources for birds. Therefore it is not surprising that more species of birds were found in the native forest than in the pine forest, which typically occurs in temperate environments as well (e.g. Von Haartman 1971, Winternitz 1976, White et al. 1996). However, Wunderle and Latta (1996) demonstrated that native pine forests in the Dominican Republic supported a more diverse avifauna than adjacent broad-leaved coffee plantations. Although we detected slightly more individual 
birds per count in the native forest than in the pine forest, the difference was not significant; thus, the overall density of birds appears to be similar in the two forests.

Of the 66 species of birds identified during this study, all are regarded as breeding residents in Trinidad (ffrench 1991); thus, neither habitat was important for Nearctic migrants, which occur in very low densities (G. Ramsawak, F.E.H. and I.S., unpublished). Although some species of birds breed throughout the year in Trinidad, the peak of breeding activity for most species coincides with the early wet season (June and July), presumably when food resources are most abundant (Snow and Snow 1964). We suspect that few species breed within the pine forest, even though the diversity and abundance of birds remained high during the peak breeding season (Figure 3). Although we did not search diligently for bird nests in either habitat, three species were found nesting in the native forest and none in the pine forest. Additional fieldwork would be required to determine which species of birds actually nest within the pine forest.

Of the two statistical tests used to compare abundance between habitats for each species, the chi-square test was considerably more powerful in detecting significant differences between the two habitats, presumably due to the low numbers of birds detected per count (usually 0,1 or 2) resulting in a large number of tied ranks for the Mann-Whitney $U$-test.

Seasonal variation in the diversity and abundance of birds was more pronounced in the pine forest, especially at the peak of the dry season in April when relatively few birds were present. During the dry season, the vegetation and soils of the pine forest are probably more exposed to water loss due to the greater amount of sunlight penetrating to the forest floor. The less humid conditions of the pine forest during the dry season probably limit the plant and arthropod food resources available to the bird community.

Unfortunately we were unable to census birds in the pine forest immediately after it was burned by fire on 8 April 1996; however, by 2 June 1996 the diversity and abundance of birds had attained a level similar to other censuses during the wet season (Figure 3). This may be attributed to the slight fire damage incurred by the pine forest canopy and the rapid regeneration and subsequent flowering of understorey herbs and shrubs, which typically occurs in Caribbean pine plantations after a fire (Kellman et al. 1987). Frequent fires may indirectly affect the pine forest bird community over a long period by inhibiting the regeneration of native broad-leaved trees.

Our data clearly indicate that the bird community of the pine forest is dominated by forest edge specialists and (to a lesser extent) habitat generalists, which is not surprising given that the pine forest had a more open canopy cover and an extensive herbaceous understorey differing little from early successional grasslands where the original forest has been cleared. The pine forest was frequently used by roosting raptors/scavengers (i.e. hawks and vultures), which are carnivorous and forage mostly in the air; in contrast, no raptors/scavengers were detected within the native forest, though they were frequently observed flying overhead. The relative abundance of nectarivores and scarcity of insectivores at the individual level within the pine forest is enigmatic; we would have predicted the opposite given the lower diversity of plant species in the pine forest. A limitation of our dietary and foraging strata categories is that they do 
not consider variation among individuals and habitats. We note, for example, that the Bananaquit (chiefly nectarivorous; ffrench 1991) foraged primarily on arthropods in the pine forest canopy, where flowering plants were conspicuously absent; in contrast, the Copper-rumped Hummingbird Amazilio tobaci foraged primarily on understorey flowers and, to a lesser extent, arthropods (pers. obs.). The more extensive undergrowth of the pine forest apparently supports more individuals per species of understorey birds. Detailed observations on resource abundance and resource use by birds in the two habitats are required to better understand the differences between the two bird communities in their dietary and foraging strata characteristics.

\section{Recommendations for management}

Jhilmit (1992) stated that the sites selected for establishing pine plantations in Trinidad are often cleared illicitly by slash-and-burn agriculturists or marijuana cultivators. We suggest that pine plantations be established only in previously cleared areas and that natural regeneration by native vegetation should be given priority, especially in sensitive areas with a threatened or specialized flora and fauna (e.g. natural savannas, most of which have been destroyed in Trinidad; Comeau 1989). Because pines grow considerably faster than the native forest trees in direct sunlight, the shade provided by pines may promote regeneration of native vegetation in the understorey (S. A. Temple, pers. comm.). Lugo (1992) demonstrated that small, unmanaged plantations of exotic tree species including the Caribbean pine - are capable of supporting a flora rich in species.

Although our results document a reduction in the diversity of birds in a pine plantation, many species of birds - particularly forest edge specialists and habitat generalists - appear capable of utilizing the food resources available in a pine plantation. Managers may increase the diversity of bird communities in pine plantations by promoting habitat heterogeneity (Cruz 1988). This may be accomplished by: (1) providing corridors of native vegetation; (2) allowing the natural vegetation to regenerate rather than removing the undergrowth mechanically, by fire or by spraying herbicides; and (3) avoiding clear-cutting by selectively harvesting pines and leaving the native broad-leaved vegetation intact.

Finally, because our study was limited to a single pine plantation, the patterns we observed in the diversity, abundance and seasonality of birds may not be the same as those in pine plantations differing in age, soils, topography, climate and intensity of management. Further studies of natural regeneration and succession in exotic pine plantations, both before (e.g. Lugo 1992) and after harvesting the pines, should be conducted to document the responses of the native flora and fauna. Ideally such studies should be conducted in all tropical countries where the Caribbean pine is introduced.

\section{Acknowledgements}

This study was inspired by the comments and discussion, primarily by P. R. Bacon, P. B. Hamel, C. K. Starr, S. A. Temple and J. M. Wunderle, on the results of a preliminary survey (conducted in early 1995) presented at the 1995 meeting of the Society of Caribbean 
Ornithology in Trinidad. Seventeen students of F.E.H.'s Tropical Ecology class (summer 1996), particularly D. R. Hardy, assisted with the vegetation sampling. G. Ramsawak (Pax Guest House) generously shared with us his knowledge of the history and birdlife of Mount Saint Benedict. E. B. Henry (Meteorological Office of Trinidad and Tobago) provided rainfall data. W. K. Hayes, J. Hinshaw (Wilson Ornithological Society), M. C. Levy (Gosse Bird Club) and R. Stacy provided pertinent literature. An anonymous referee supplied excellent suggestions for improving the manuscript. We thank all of these individuals for their assistance.

\section{References}

American Ornithologists' Union. (1983) Check-list of North American birds, sixth edition. Washington, D.C.: American Ornithologists' Union.

Anon. (1989) The national forest resources plan. Port of Spain, Trinidad: Forestry Division, Ministry of Environment and National Service.

Anon. (1990) Statistix manual. St Paul, Minn.: Analytical Software.

Anoruo, A. O. and Berlyn, G. P. (1993) Caribbean pine in sustainable tropical forestry: distribution, taxonomy, ecology, biotechnology and silvics. J. Sust. For. 1: 1-23.

Bandeira, A. G. and Souza, P. C. S. (1982) Influência do pinheiro (Pinus caribaea) sobre a fauna do solo na Amazônia. Bol. Museu Para Emílio Goeldi Zool. Belem 114: 1-13.

Barbour, M. G., Burk, J. H. and Pitts, W. D. (1980) Terrestrial plant ecology. Menlo Park, Calif.: Benjamin/Cummings.

Beard, J. S. (1946) The natural vegetation of Trinidad. Oxford For. Mem. 20: 1-152.

Beebe, W. (1952) Introduction to the ecology of the Arima Valley, Trinidad, B. W. I. Zoologica 37: 157-183.

Berridge, C. E. (1981) Climate. Pp. 2-12 in St. G. C. Cooper and P. R. Bacon, eds. The natural resources of Trinidad and Tobago. London: Edward Arnold.

Bibby, C. J., Burgess, N. D. and Hill, D. A. (1992) Bird census techniques. London: Academic Press.

Brash, A. R. (1987) The history of avian extinction and forest conversion on Puerto Rico. Biol. Conserv. 39: 97-111.

Brown, S. and Lugo, A. E. (1990) Tropical secondary forests. J. Trop. Ecol. 6: 1-32.

Brown, S., Lugo, A. E. and Chapman, J. (1986) Biomass of tropical tree plantations and its implications for the global carbon budget. Can. J. For. Res. 16: 390-394.

Comeau, P. L. (1989) Savannas in Trinidad. Living World (J. Trin. Tob. Field Nat. Club) 19891990: 5-8.

Comeau, P. L. (1991) Geological events influencing natural vegetation in Trinidad. Living World (J. Trin. Tob. Field Nat. Club) 1991-1992: 29-38.

Cruz, A. (1987) Avian community organization in a mahogany plantation on a Neotropical island. Carib. J. Sci. 23: 286-296.

Cruz, A. (1988) Avian resource use in a Caribbean pine plantation. J. Wildl. Mgmt. 52: $274-279$.

Evans, J. (1982) Plantation forestry in the tropics. Oxford: Clarendon Press.

Falkenberg, W. C., Robinson, C. and Maynard, J. K. (1983) Will the forest birds of Jamaica live in pine plantation? Gosse Bird Club Broadsheet 40: 1-7.

ffrench, R. (1991) A guide to the birds of Trinidad and Tobago. Second edition. Ithaca, N.Y.: Cornell University Press.

ffrench, R. and Bacon, P. (1992) Nature trails of Trinidad, revised edition (V. Quesnel, ed.) Port of Spain, Trinidad: SM Publications.

Gotelli, N. J. and Graves, G. R. (1990) Body size and the occurrence of avian species on land-bridge islands. J. Biogeogr. 17: 315-325. 
Graves, G. R. and Gotelli, N. J. (1983) Neotropical land-bridge avifaunas: new approaches to null hypotheses in biogeography. Oikos 41: 322-333.

Hutto, R. L., Pletschet, S. M. and Hendricks, P. (1986) A fixed-radius point count method for nonbreeding and breeding season use. Auk 103: 593-602.

Jhilmit, S. (1992) Manual for the production of pine (Pinus caribaea var. hondurensis) in Trinidad. Port of Spain, Trinidad: Ministry of Agriculture, Land and Marine Resources.

Kellman, M., Miyanishi, K. and Hiebert, P. (1987) Nutrient sequestering by the understorey strata of natural Pinus caribaea stands subject to prescription burning. For. Ecol. Mgmt. 21: 57-73.

Lackhan, N. P. (1972) Pinus caribaea (var. hondurensis) in Trinidad and Tobago. Port of Spain: Forestry Division, Ministry of Agriculture, Lands and Food Production.

Lackhan, N. P. (1973) Pinus caribaea in Trinidad and Tobago. J. Agr. Soc. Trin. Tob. 73: 190222.

Lemmon, P. E. (1957) A new instrument for measuring forest overstory density. J. For. 55: $667-668$.

Lugo, A. E. (1992) Comparison of tropical tree plantations with secondary forests of similar age. Ecol. Monogr. 62: 1-41.

Murphy, W. L. (1995) A birder's guide to Trinidad and Tobago, second edition. Parkersburg, W.Va.: Peregrine Enterprises.

Perfecto, I., Rice, R. A., Greenberg, R. and Van der Voort, M. E. (1996) Shade coffee: a disappearing refuge for biodiversity. BioScience 46: 598-608.

Perry, J. P., Jr. (1991) The pines of Mexico and Central America. Portland, Ore.: Timber Press.

Ralph, C. J. and Scott, J. M., eds. (1981) Estimating numbers of terrestrial birds. Stud. Avian Biol. 6: 1-630.

Ralph, C. J., Sauer, J. R. and Droege, S., eds. (1995) Monitoring bird populations by point counts. Pacific Southwest Research Station, Forestry Service, U.S. Department of Agriculture (Gen. Techn. Rep., PSW-GTR-149: 1-187).

Ramsawak, G. (1996) The trail guide to Mt St. Benedict. Tunapuna, Trinidad: Pax Guest House, Mt St. Benedict Ltd.

Snow, D. W. (1985) Affinities and recent history of the avifauna of Trinidad and Tobago. Orn. Monogr. 36: 238-246.

Snow, D. W. and Snow, B. K. (1964) Breeding seasons and annual cycles of Trinidad land birds. Zoologica 49: 1-39.

Torres, R., Pino, G. and Smith, N. V. (1978) Correlación entre la cobertura de bosque de Pinus caribaea var. hondurensis y la diversidad del estrato herbáceo y de la microfauna de arthrópodos. Mem. Soc. Cienc. Nat. La Salle, Caracas 38(109): 9-18.

Trinidad and Tobago Government (1970) [Map of] Trinidad. Sheet 13, 1:25,000, second edition. Port of Spain, Trinidad: Trinidad and Tobago Government.

Trinidad and Tobago Government (1978) [Map of] Trinidad. Sheet 14. 1:25,000, second edition. Port of Spain, Trinidad: Trinidad and Tobago Government.

Verner, J. (1985) Assessment of counting techniques. Pp. 247-302 in R. F. Johnston, ed. Current Ornithology, 2. New York: Plenum Press.

Vidakovic, M. (1991) Conifers: morphology and variation, second edition. Zagreb, Croatia: Graficki zavod Hrvatske.

Von Haartman, L. (1971) Population dynamics. Pp. 391-459 in D. S. Farner and J. R. King, eds. Avian biology, 1. New York: Academic Press.

White, D. H., Kepler, C. B., Hatfield, J. S., Sykes, P. W., Jr. and Seginak, J. T. (1996) Habitat associations of birds in the Georgia Piedmont during winter. J. Field Orn. 67: 159-166.

Wiedenfeld, D. A. (1991) Tropical bird species numbers in second-growth vs primary forest habitats at large scales. Orn. Neotrop. 2: 40-43.

Winternitz, B. L. (1976) Temporal change and habitat preference of some montane breeding birds. Condor $78: 383-393$. 
Wunderle, J. M., Jr. (1994) Census methods for Caribbean land birds. U.S. Department of Agriculture Forestry Service, South Fork Experimental Station (Gen. Techn. Rep. SO-98: 1-26).

Wunderle, J. M., Jr. and Latta, S. C. (1996) Avian abundance in sun and shade coffee plantations and remnant pine forest in the Cordillera Central, Dominican Republic. Orn. Neotrop. 7: 19-34.

Zar, J. H. (1984) Biostatistical analysis, second edition. Englewood Cliffs, N.J.: Prentice-Hall.

\section{FLOYD E. HAYES}

Department of Biology, Caribbean Union College, P.O. Box 175, Port of Spain, Trinidad and Tobago. Present address: Department of Life Science, University of the West Indies, St. Augustine, Trinidad and Tobago

ISHMAELANGELO SAMAD

El Tucuche Hiking Lodge and Nature Retreat, Loango Village, Maracas, Trinidad and Tobago 